\title{
Dynamic Compensation for Impact-Based Grain Flow Sensor
}

\author{
Junwan $\mathrm{Hu}^{1, *}$, Changlai Gong ${ }^{1}$, and Zhigang Zhang ${ }^{2}$ \\ ${ }^{1}$ Electronical Information Engineering College, JiaYing University, Meizhou 514015, China \\ hujunwan@jyu.edu.cn \\ ${ }^{2}$ Key Laboratory of Key Technology on Agricultural Machine and Equipment \\ of Ministry of Education, South China Agricultural University, \\ Guangz hou 510642, China
}

\begin{abstract}
The impact-based grain flow sensor is widely used in the combine harvester yield monitor systems to measure the crop yield .but its accuracy wrecked by false signal of excessive natural vibration. A step response experiment was carried to identify the math function of the sensor. Experiment found that the math function of the sensor was a second-order vibration system, its Damping Ratio was 0.037 , and its natural frequency was $125 \mathrm{~Hz}$, the overshoot reached $20 \%$. To reduce the effect of the overshoot and natural vibration signal on the measurement accuracy, a dynamic compensation algorithm was designed with the series correction method. Step response experiment showed that the overshoot of the compensated sensor was reduced from $20 \%$ to $2 \%$.
\end{abstract}

Keywords: impact-based grain flow sensor, system identification, transfer function, dynamic compensation.

\section{Introduction}

As an important element of the combine yield monitor system The impact-based grain flow sensor measure the grain flow of the harvester by detecting the impact of grain. With the characteristics of simple structure, and low cost, the impact-based grain flow sensor is widely used in the combine harvester yield monitor systems to measure the crop yield [1-11], But its accuracy was influent by vibration and tilt [12-15].

With a high velocity, the grain jet out of the crop outlet of the combine harvester, and impact the detect plate of the sensor and reflect quickly. The yield monitor system acquires the impact force and calculates the grain flow rapidly. But, after impacting, a false signal last a while by overshoot and nature vibration.

A Damping material was adopted to enhance Damping Ratio of sensor, and then eliminate the affection of overshoot and nature vibration [16-18], but reduce the sensitivity.

To investigate the dynamic characteristic, a step response experiment was carried to identify the math function of the sensor. And a dynamic compensation algorithm was designed with the series correction method in this paper.

* Lecturer, Research on agricultural automation. 


\section{The Impact-Based Grain Flow Sensor}

As show in Fig. 1, the single plate impact-based grain flow include bracket. elastic body, impact plate.

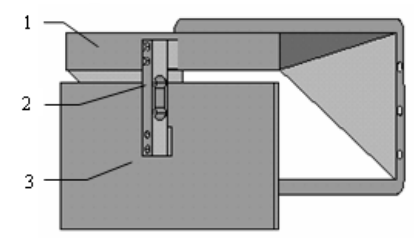

Fig. 1. Structure of impact-based grain flow sensor

The impact plate is made of a Plexiglas board with the dimensions $260 \mathrm{~mm}$ $\times 120 \mathrm{~mm} \times 4 \mathrm{~mm}$. It fixed at the end of elastic body which bean made of a hard aluminum-Alloy with the dimensions of $80 \mathrm{~mm} \times 12 \mathrm{~mm} \times 12 \mathrm{~mm}$, and 2 parallel through-holes in its center.

As show in the Fig.2, a Wheatstone bridge with 4 electrical-resistance strain gages with $320 \Omega$ bean pasted at the most sensitive surface of the elastic body.
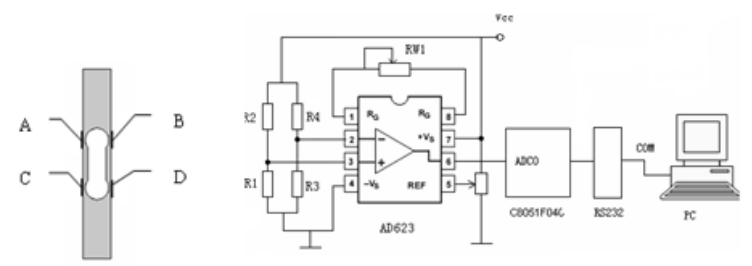

Fig. 2. Data acquiring circuit for sensor

The grain impact the plate of the sensor when the combine harvester working, and then, the output signal of the sensor have a liner relationship with the impact force. The signal is amplified from $0 \sim 5 \mathrm{mV}$ into $0 \sim 5 \mathrm{~V}$ by a AD623 instrumentation amplify circuit. In this way, we can calculate the grain flow by the output voltage of the sensor.

The output voltage of the sensor can be acquired by a embed ADC of C8051F040 MCU, and send to a computer by RS232 Bus.

\section{System Identifications}

The impact-based grain sensor belong A Mass-elastic-Damper system. According to the basic theory of automatic, the impact-based grain flow sensor is a typical attenuate vibration system of second order, its transfer function should be: 


$$
G(s)=\frac{\omega_{n}^{2}}{S^{2}+2 \xi \omega_{n} S+\omega_{n}^{2}}
$$

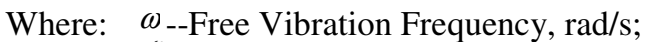

$\xi$--Damping Ratio, No dimension.

The parameter of transfer function can be identified by Step Response test.

\subsection{Step Response Test}

As the grain flow sensor belongs a Mass elastic system, the nature frequency could be changed by put a mass-block load. So step signal can be conducted by unload a pre-load suddenly.

As show in Fig.3, fix the sensor in level, and hang a $2 \mathrm{~kg}$ mass-block as pre-load with a slender thread. Adjust the zero point; make sure the output of the sensor is $0 \mathrm{~V}$.

The step signal happened by unload the preload with a pair of shears suddenly.

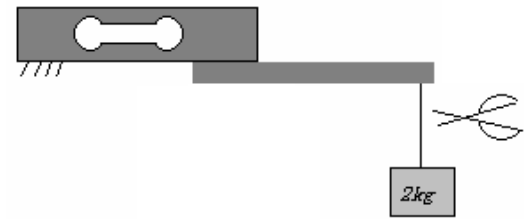

Fig. 3. Step load method

\subsection{Test Results}

The output voltage under step signal excitation was acquired by C8051F040, and recorded by PC. As show in Fig.4, the step response curve indicates that the grain flow sensor is a typical Second Order System.

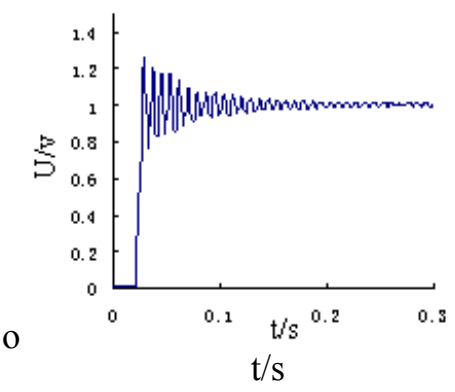

Fig. 4. Step response of the sensor

According to the basic theory of automatic, there is: 


$$
\begin{gathered}
\omega_{d}=\omega_{n} \sqrt{1-\xi^{2}} \\
\xi \omega_{n}=\frac{1}{T} \ln \left(\frac{A_{1}}{A_{2}}\right)
\end{gathered}
$$

Where $\omega_{d}--$ angular frequency under damped vibration, rad/s;

T--period of damped vibration, $s$;

$A_{1}$--the first peak voltages, $\mathrm{V}$;

$A_{2}--$ the second peak voltages, $V$.

From the curve, $\mathrm{T}=0.008 \mathrm{~s}, A_{1}=0.265 \mathrm{v}$,

$A_{2}=0.210 \mathrm{v}, \cdot \omega_{d}=2 \pi \frac{1}{T}=785 \mathrm{rad} / \mathrm{s}$ Then $\omega_{n} \approx 785 \mathrm{rad} / \mathrm{s}, \xi \approx 0.037$, the

transfer function of the grain flow sensor is:

$$
G(s)=\frac{616225}{S^{2}+58 S+616225}
$$

The overshoot reached $20 \%$, the accuracy of the sensor could be damage by overshoot and free vibration.

\section{Dynamic Compensation}

\subsection{Basic Theory of Compensation}

For a second order system with small Damping Ratio, it's necessary to compensate the Damping Ratio up to 0.6 0.7 for the stability. Take an additional Damping material can improve the Damping Ratio in some degree, but it is not enough. Series/parallel correction methods are widely used in sensor dynamic compensation [15-16].

If the original transfer function is $G(s)$, the series correction system is $G_{c}(s)$, the after correction system should be:

$$
G^{\prime}(s)=G(s) G_{C}(s)
$$

According to the formula (1), If the appropriate Damping ratio is 0.6, and keep the nature frequency, then the ideal corrected system should be:

$$
G^{\prime}(s)=\frac{616225}{S^{2}+942 S+616225}
$$


By Matlab simulate, we got the step response curve of the original system and the corrected system. As show in Fig.5, the simulation result showed that the overshoot of the corrected system can be limited significantly. The corrected system should keep more stability.

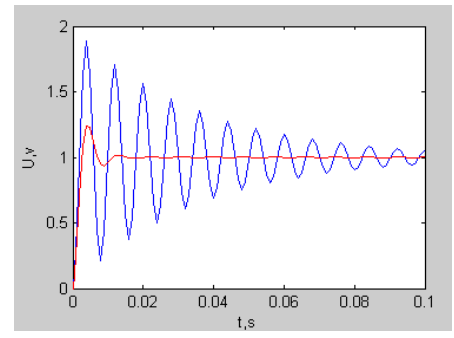

Fig. 5. Step response by simulation

According to the formula (5)and (6), the series correction system should be:

$$
G_{c}(s)=\frac{S^{2}+58 S+616225}{S^{2}+942 S+616225}
$$

\subsection{Compensation Algorithm}

It is difficult to reality the formula (7) by create a suitable hardware circuit. But it is easy for software. For that, the correction system must describe in discrete transfer function.

Make a Z Transform by Matlab function named C2D with a $0.001 \mathrm{~s}$ sampling period, the formula (7) transform into discrete transfer function:

$$
G_{c}(z)=\frac{0.6849 z^{2}-0.9246 z+0.619}{z^{2}-1.01 z+0.03898}
$$

According to formula (8), If the input of the correction system is $\mathrm{x}$, the output is $\mathrm{y}$, the second order difference equations of the correction system is:

$$
\begin{aligned}
& y^{*}(t+2 T)-1.01 y^{*}(t+T)+0.03898 y^{*}(t) \\
& =0.6849 x^{*}(t+2 T)-0.9246 x^{*}(t+T)+0.619 x^{*}(t)
\end{aligned}
$$

Where $\mathrm{y}^{*}$-- discrete value of output variable $\mathrm{y}$;

$\mathrm{x}^{*}$-- discrete value of input variable $\mathrm{x}$;

$\mathrm{T}$-sampling period, $0.001 \mathrm{~s}$.

According to formula (9), get the iterative equation: 


$$
\begin{aligned}
& y^{*}(k+2)=0.6849 x^{*}(k+2)-0.9246 x^{*}(k+1) \\
& +0.619 x^{*}(k)+1.01 y^{*}(k+1)-0.03898 y^{*}(k)
\end{aligned}
$$

Where $\mathrm{k}$-positive integer;

initial condition of the iterative equation:

$$
\begin{array}{ll}
x^{*}(0)=1 & y^{*}(0)=0 \\
x^{*}(1)=1 & y^{*}(1)=0 \ldots
\end{array}
$$

Finally, program the compensation formula (10) and (11) into fire ware of C8051F040.

\subsection{Compensation Result}

Step response test carried again after compensation. Show as at Fig.6.

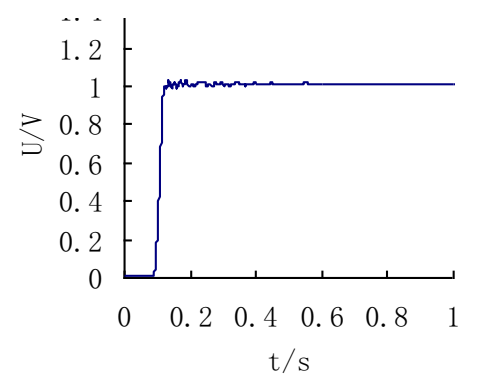

Fig. 6. Step response after compensation

The response curve of the compensated showed that the overshoot been limited from $20 \%$ to $2 \%$, the dynamic characteristics improved significantly.

\section{Conclusion}

The impact-based grain flow sensor system was identified by a step response test. The step response curve indicated that the transfer function of the grain flow sensor is a under Damping second-order system with $125 \mathrm{~Hz}$ free frequency and 0.037 damped Ratio.

A compensation algorithm was introduced to improve the dynamic characteristics without change the real damped Ratio. After compensation, the overshoot limited from $20 \%$ to $2 \%$.The dynamic characteristics have been improved significantly.

\section{References}

1. Luo, X., Zhang, Y., Zhou, Z.: Research progress in farming information acquisition technique for precision agriculture. Transactions of Agricultural Engineering 22(1), 167-173 (2006) (in Chinese) 
2. Li, M.: The Technique of Crop Yield Monitor and Key Equipment. Agriculture Network Information (S1), 34-38 (2004) (in Chinese)

3. Reyns, P., Missotten, B., Ramon, H., et al.: A Review of Combine Sensors for Precision Farming. Precision Agriculture (3), 169-182 (2002) (in Chinese)

4. Sun, Y., Wang, M., Ma, D., Huang, J.: Experimental Research on Grain flow measurement System Using an Impact sensor. Transactions of Agricultural Machinery (04), 48-50 (2001) (in Chinese)

5. Chen, S., Zhang, W., Li, X., Li, M., Huang, J.: Experiment Research of Grain Mass Flow Sensor Based on Impact. Transactions of Agricultural Machinery (02), 82-84 (2005) (in Chinese)

6. Lee, C.K., Iida, M., Kaho, T., et al.: Development of impact type sensor for heading feeding combine. Journal of the JSAM 62(4), 81-88 (2000) (in Japanese)

7. Shoji, K., Kawamura, T., Horio, H.: Impact-based grain yield sensor with compensation for vibration and drift. Journal of the JSAM 64(5), 108-115 (2002) (in Japanese)

8. Chaplin, J., Hemming, N., Hetchler, B.: Comparison of impact plate and torque-based grain mass flow sensors. Transactions of the American Society of Agricultural Engineers 47(4), 1337-1345 (2004)

9. Schrock, M.D., Oard, D.L., Eisele, E.L., et al.: A diaphragm impact sensor for measuring combines grain flow. Applied Engineering in Agriculture 15(6), 639-642 (1999)

10. Strubbe, G., Missotten, B., De Baerdemaeker, J.: Mass flow measurements with a curved plate at the exit of an elevator. In: Proceedings of the 3rd International Conference on Precision Agriculture, USA, (1996)

11. Vansichen, R., De Baerdemaeker, J.: An impact type flow rate sensor for combines. In: International Conference on Agricultural Engineering, Uppsala, Sweden, pp. 15-16 (1992)

12. Arslan, S., Colvin, T.S.: Laboratory performance of a yield monitor. Applied Enginering in Agriculture 15(03), 189-195 (1999)

13. Burks, T.F., Shearer, S.A., Fulton, J.P., et al.: Combine yield monitor test facility development and initial monitoring test. Applied Engineering in Agriculture 19(01), 5-12 (2003)

14. Fulton, J.P., Sobolik, C.J., Shearer, S.A., et al.: Grain yield monitor flow sensor accuracy for simulated varying field slopes. Applied Engineering in Agriculture 25(1), 15-21 (2009)

15. Grisso, R.D., Jasa, P.J., Schroeder, M.A., et al.: Yield monitor accuracy successful farming magazine case study. Applied Engineering in Agriculture 18(02), 147-151 (2002)

16. Zhou, J., Zhou, G., Miao, Y., Liu, C.: Damping Design of Impact-based Grain Yield Sensor. Transactions of the Chinese Society for Agricultural Machinery 36(11), 121-123 (2005)

(in Chinese)

17. Tu, J., Miao, Y., Zhao, S., et al.: Damping desige and signal proscession of the yield sensor on grain combine. In: Progress of Information Technology in Agriculture, Proceedings of the 4th International Symposium on Intelligent Information Technology in Agriculture(ISIITA), Beijing (2007)

18. Zhou, J., Liu, C.: Signal processing method for impact-based grain mass flow sensor with parallel beam load cell. Transactions of the Chinese Society of Agricultural Engineering 24(1), 183-187 (2008) (in Chinese)

19. Chen, S., Yang, H., Li, Y., Hu, J., Zhang, L.: Experiment of Dual-plates Differential Impact-based Grain Flow Sensor. Transactions of the Chinese Society for Agricultural Machinery 41(08), 172-173 (2010) (in Chinese) 